\title{
Removing Impulse Bursts from Images by Training-Based Filtering
}

\author{
Pertti Koivisto \\ Department of Mathematics, Statistics, and Philosophy, University of Tampere, Finland \\ Institute of Signal Processing, Tampere University of Technology, Tampere, Finland \\ Email:pertti.koivisto@tut.fi
}

Jaakko Astola

Institute of Signal Processing, Tampere University of Technology, Tampere, Finland

Email: jaakko.astola@tut.fi

\section{Vladimir Lukin}

Department of Receivers, Transmitters, and Signal Processing, National Aerospace University

(Kharkov Aviation Institute), Kharkov, Ukraine

Email:lukin@xai.kharkov.ua

\section{Vladimir Melnik}

Institute of Signal Processing, Tampere University of Technology, Tampere, Finland

Email:vladimir.melnik@nokia.com

\section{Oleg Tsymbal}

Department of Receivers, Transmitters, and Signal Processing, National Aerospace University

(Kharkov Aviation Institute), Kharkov, Ukraine

Email:dmb@ire.kharkov.ua

Received 18 March 2002 and in revised form 15 September 2002

\begin{abstract}
The characteristics of impulse bursts in remote sensing images are analyzed and a model for this noise is proposed. The model also takes into consideration other noise types, for example, the multiplicative noise present in radar images. As a case study, soft morphological filters utilizing a training-based optimization scheme are used for the noise removal. Different approaches for the training are discussed. It is shown that these techniques can provide an effective removal of impulse bursts. At the same time, other noise types in images, for example, the multiplicative noise, can be suppressed without compromising good edge and detail preservation. Numerical simulation results, as well as examples of real remote sensing images, are presented.
\end{abstract}

Keywords and phrases: impulse burst removal, burst model, soft morphological filters, training-based optimization.

\section{INTRODUCTION}

Remote sensing images are usually formed on board an aircraft or spaceborne carrier where sensors and primary signal processing devices are installed [1]. Then, the images are transferred to one or a few on-land remote sensing dataprocessing centers, where they are subject to visualization, analysis, filtering, interpretation, and so forth. For transferring the remote sensing data, the standard or special communication channels are used and, since images are often encoded and then decoded, impulsive noise may be observed in images [2].
In many practical situations, the probability of spikes is low and two or more neighboring pixels are very seldom corrupted by impulsive noise. In other words, the spikes possess an approximately spatially invariant characteristic. Many efficient and robust filtering algorithms have been already proposed to remove spikes that fulfill the aforementioned model assumptions $[3,4,5]$. However, these assumptions are not valid in some practical situations.

For example, interference may occur when the remote sensing data is transferred using analog signal communication channel and the widely used automatic picture transmission format [6]. This interference can be long term and so 


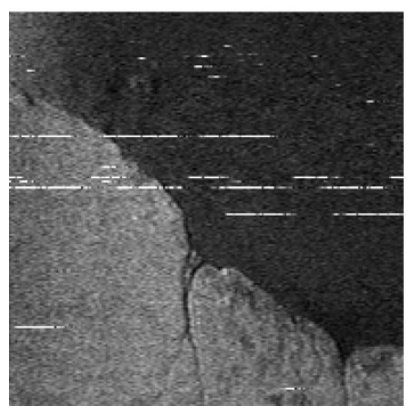

(a)

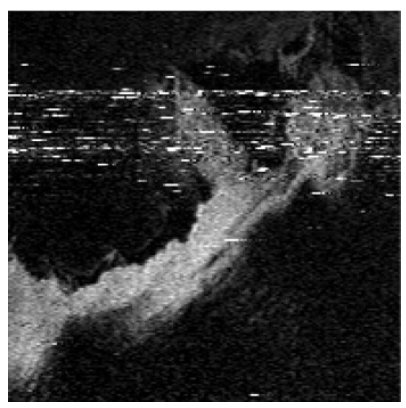

(b)

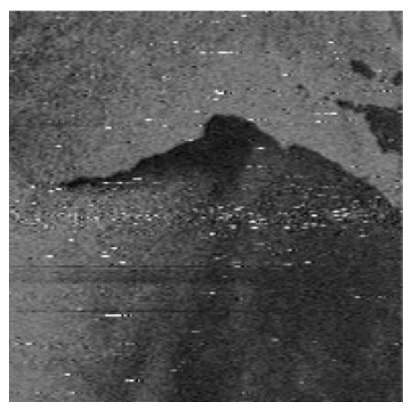

(c)

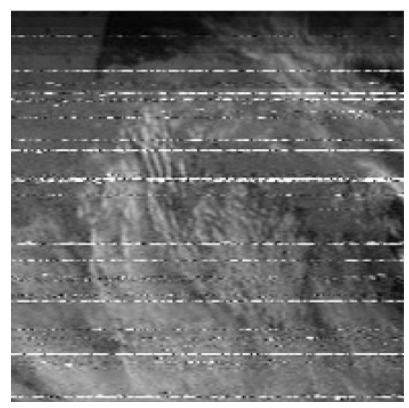

(d)

FIGURE 1: Four $192 \times 192$ parts of the original satellite images. Images (a), (b), and (c) are radar images and image (d) is an optical image.

intensive that it corrupts several consecutive image pixels in one or more rows following each other. (In this paper, we assume that images are transferred rowwise. Naturally, similar effects can also be observed and methods similar to those examined in this paper can be applied if images are transferred columnwise.) Such situations may happen if the receiver input and circuitry are not well protected against intensive interference or if in the neighborhood of the remote sensing data processing center, there are some electromagnetic wave irradiation sources operating in the frequency band which overlaps with the communication channel waveband.

Real life illustrations of what happens in this case with satellite images are presented in Figure 1. As can be seen, different amounts of horizontal impulse bursts appear in these images. This kind of bursts appearing as line-type noise considerably decrease the image quality. Hence, these bursts must be removed. This is, however, not a typical and easy task for the majority of commonly used filters.

It may seem that impulse bursts and multiplicative noise can be simultaneously removed by some robust scanning window filter. However, experiments show that such scanning window filters do not produce good results. The robust filters (e.g., the median filter) remove impulse bursts but at the same time they usually destroy details, small size objects, and texture too heavily. Other filters, for example, the centerweighted median and the modified sigma filter that was introduced [7] to filter images that are corrupted both by multiplicative and by impulsive noise, are not robust enough and thus a lot of impulse bursts may remain in the images after the filtering.

Another possibility might be first to detect the pixels corrupted by impulse bursts and then to replace the corresponding values by new values, usually by taking in some way into account the neighboring pixel values for which the bursts have not been detected. However, in general, spike detection methods (e.g., $[8,9])$ do not perform well in this task. The reason why these methods fail is that they have been designed to detect either isolated impulses or bursts whose characteristics differ from the characteristics of the considered impulse bursts.

One more possibility is to utilize training-based filter design. For example, Koivisto et al. [10] have shown that training-based optimized soft morphological filters are able to remove line-type noise efficiently. The designed filters could also remove line-type noise with horizontal or almost horizontal orientations. As this noise in a certain sense corresponds to the impulse bursts that we are considering, it is reasonable to expect that soft morphological filters being trained for the removal of impulse bursts are able to perform well for image recovery in our case.

There are also some differences between the task considered here and the design task studied by Koivisto et al. in their paper. First, the impulse bursts differ from the line-type noise since the former one has more complicated and random behavior. Second, besides impulse bursts, the remote sensing images usually contain other types of noise as well. For instance, the radar images are characterized by the presence of multiplicative noise [4]. Hence, the training task is now much more complicated.

In this paper, we analyze the properties of impulse bursts in remote sensing images and propose a model for this noise. The model also takes into consideration other noise types present in images. This model is then used in the formation of the training images used in the optimization of the soft morphological filters. In addition, different approaches for the training and filtering are discussed. Finally, numerical simulation results as well as test image and real remote sensing image examples are presented.

\section{IMPULSE BURSTS IN REMOTE SENSING IMAGES}

To get an idea what the impulse bursts are, we first analyze some real remote sensing images. The images for which the impulse bursts are observed are transferred from such low altitude satellites as NOAA (usually two satellites are operating with carrier frequencies $137.5 \mathrm{MHz}$ and 137.62 MHz), Meteor (137.85 MHz), Sich (137.4 MHz), and Okean $(137.4 \mathrm{MHz})$. The probability of the impulse bursts in the received data was the largest for the descending parts of the satellite orbits (just before the satellites escape under the horizon).

The radio frequency carrier is frequency-modulated (FM) with a deviation of $\pm 17 \mathrm{kHz}$ for the NOAA and Meteor satellites. For the Sich and Okean satellites, the FM deviation 


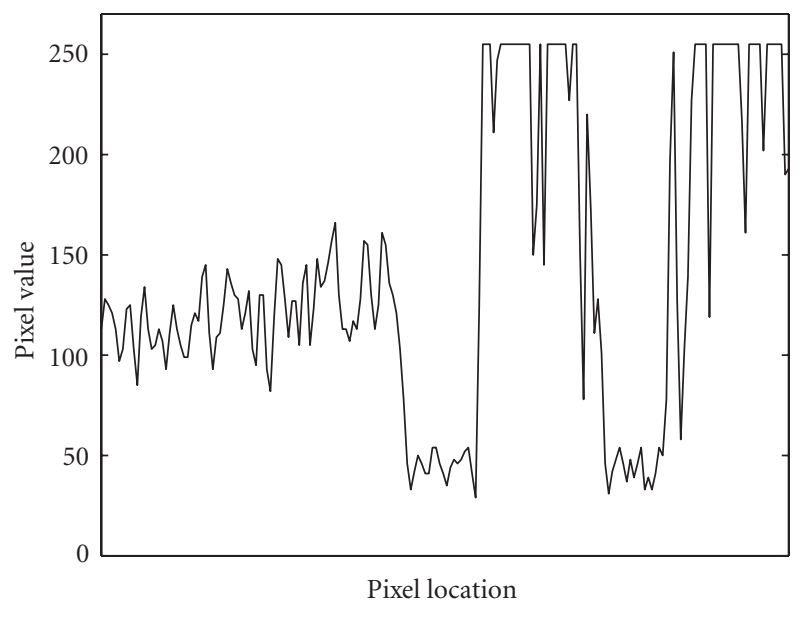

Figure 2: Row 98 in the image in Figure 1a.

is slightly smaller. All aforementioned satellites use, as one possible mode, the AM APT (automatic picture transmission) format, for which the image information is contained in the amplitude modulation of $2400 \mathrm{~Hz}$ subcarrier. More detailed information can be found, for example, in [6].

For the reception of the signals carrying the image information, we have to use a converter for the microwave frequency with an input at $137 \mathrm{MHz}$. The received images can then be decoded, and the resulting bitmap images (in some cases, these are images formed in different wavebands including radar, visible optical, and infrared) can be stored, processed, and visualized by standard programs. The modulation and decoding modes are not very well protected against interference that may be present in the $137 \mathrm{MHz}$ band. This interference can radically degrade the structure of the received signal. Hence, decoding errors appearing as impulse bursts may occur.

Four $192 \times 192$ parts of real satellite images are presented in Figure 1. As can be seen, several fragments in many rows are corrupted by impulse bursts, and the lengths of such fragments are rather different. Sometimes such fragments occur in two consecutive rows. It can also be observed that in some pixels of the considered fragments, the values are maximal (i.e., 255 in the 8-bit representation used) while most of the pixel values in the fragments differ from 255 but still remain "impulsive" with respect to the values that can be predicted for the satellite images from their local analysis. Similar effects can also be observed with the minimal value (i.e., 0 ).

\section{PROPERTIES OF IMPULSE BURSTS}

In order to make an adequate model for a test remote sensing image, we studied the properties of the real satellite images in detail. More precisely, the statistical characteristics of impulse bursts and the signal sample behavior were carefully studied row by row for the rows containing bursts. Examples of such rows are given in Figures 2 and 3. As can be seen, row 98 in Figure 2 contains two short-time impulse bursts located at the right part of the row. The presence of multiplicative

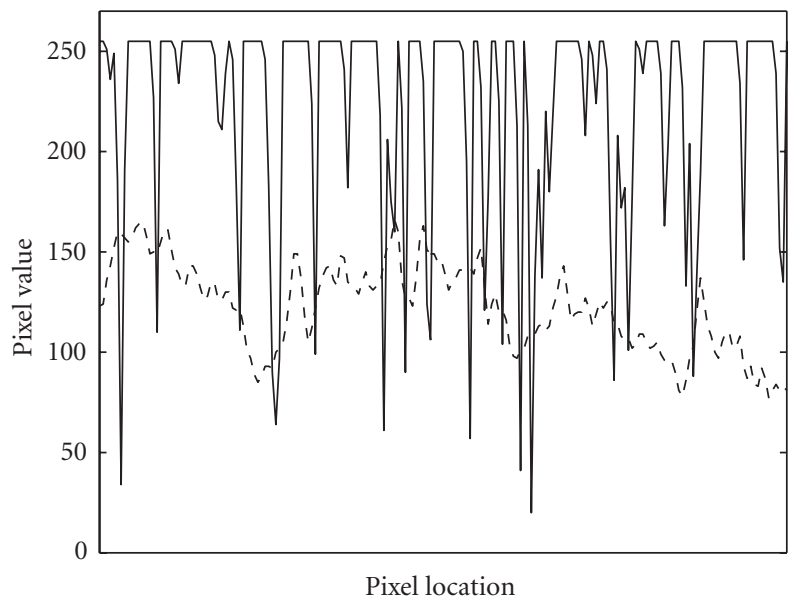

Figure 3: Rows 167 (dashed) and 168 (solid) in the image in Figure 1d.

noise in this radar image is also clearly seen. For comparison, row 168 in Figure 3 is practically fully corrupted by a long-term burst while row 167 in Figure 3 does not contain impulse bursts and shows a typical cross section of the optical image in Figure 1d. Altogether, more than 50 impulse bursts taken from the images in Figure 1 were analyzed.

It was found out that the means of the impulse bursts were usually larger than the mean of the pixels not corrupted by bursts. Visually, this means that the observed impulse bursts mainly appear as light horizontal distortions that may corrupt even two or more neighboring rows. Usually, the mean of the values of an impulse burst is larger than 160 but smaller than 190.

Most impulse bursts also contain a periodical (quasisinusoidal) component and a random noise component. Using spectral analysis of short-term time series [11], we found out that one harmonic component was practically always much larger than the other harmonic components. Hence, the latter ones can be considered as noise. After this, it was possible to estimate the amplitude and the normalized circular frequency of the dominant sinusoidal component. According to our experiments, the amplitude was from 50 to 90 while the circular frequency varied from 0.3 to 1.0 . The phase of the dominant sinusoidal component seemed to be random.

When the values for the amplitude and frequency had been estimated, it was possible to evaluate the power of the other spectral components and, using Parseval's theorem [11], to estimate the variance (or the standard deviation) of the random noise component. The estimates obtained for the standard deviation were from 22 to 40 . It was also possible to consider the noise component as consisting of independent and identically distributed (i.i.d.) random variables.

Some cutoff effects were observed as well. That is, there may be several pixels in a row having values equal to 255 , as can also be seen in Figures 2 and 3. This means that the estimated mean values for the impulse bursts may be slightly less than they would be without the cutoff effect. Obviously, this effect can be easily simulated in our artificial test image. 
Finally, the probability that a pixel belongs to an impulse burst was estimated. For the considered images, this probability was from 0.01 to 0.05 . The length of the bursts was random. In the considered images, the shortest bursts were only a few pixels long while the longest ones contained hundreds of pixels.

\section{NOISE MODEL}

All aforementioned properties of impulse bursts have been taken into account when generating the noise model for the test images. As a case study, the model is generated for side-look aperture radar (SLAR) images. Hence, the images are supposed to contain multiplicative noise with Gaussian probability density function with 1.0 as the mean $[1,4]$. Empirical tests confirm this assumption for the test images. In our cases, the estimated relative variance $\sigma_{\mu}^{2}$ of the multiplicative noise varied from 0.015 to 0.055 . Since the images are transferred as one-dimensional arrays, the noise model is also presented for onedimensional array. More precisely, our noise model is the following.

First, a Markov chain with two states is used to determine which samples (fragments) belong to impulse bursts [12]. The transition probability from "no-burst state" to "burst state" is $p$, and the transition probability from "burst state" to "no-burst state" is $q$. The values of these variables should be based on the estimated percentage of the pixels corrupted by impulse bursts.

If a sample does not belong to an impulse burst, then it is corrupted by the aforementioned multiplicative noise in the usual way. That is, the (corrupted) sample value $\hat{X}_{j}$ is given by

$$
\widehat{X}_{j}=\mu_{j} X_{j}
$$

where $X_{j}$ is the corresponding value of the original signal and $\mu_{j}$ is the multiplicative noise component having relative variance $\sigma_{\mu}^{2}$ (and mean equal to 1.0). If we do not want to add multiplicative noise (e.g., the image is a satellite image that is already corrupted by multiplicative noise), we can set $\sigma_{\mu}^{2}=0$.

On the other hand, if the $j$ th sample belongs to the $k$ th impulse burst, then the (corrupted) sample value $\hat{X}_{j}$ is obtained using the formula

$$
\hat{X}_{j}=\operatorname{round}\left\{\alpha_{k}+\beta_{k} \sin \left[\left(j-l_{k}\right) \omega_{k}+\varphi_{k}\right]+\xi_{j}\right\},
$$

where $l_{k}$ denotes the index of the leftmost sample in the burst (i.e., the starting index of the burst), $\alpha_{k}$ is the average level of the impulsive noise in the burst, $\beta_{k}$ and $\omega_{k}$ are the amplitude and the circular frequency of the harmonic component of the burst, respectively, $\varphi_{k}$ denotes the phase of the harmonic component of the burst, and $\xi_{j}$ is the fluctuating noise component of the burst. The parameters $\alpha_{k}, \beta_{k}, \omega_{k}$, and $\varphi_{k}$ are random variables with uniform distribution from the intervals ${ }^{1} \mathscr{A} \subseteq[0,255], \mathscr{B} \subseteq[0,255], \mathscr{O} \subseteq[0,2 \pi[$, and ] $-\pi, \pi]$, respectively. The noise component $\xi_{j}$ is a random variable with Gaussian probability density function with zero mean and standard deviation $\sigma_{k}$, where $\sigma_{k}$ is a random variable with uniform distribution from the interval $\mathscr{Y} \subseteq[0, \infty[$. Rounding is to the nearest nonnegative integer less than or equal to 255 .

Hence, the parameters $\alpha_{k}, \beta_{k}, \omega_{k}, \varphi_{k}$, and $\sigma_{k}$ change from burst to burst but are common to all pixels in some burst. The parameter $\xi_{j}$ varies from pixel to pixel. The parameters are modeled as random variables to simulate the random behavior of the impulse bursts in the real satellite images.

In order to apply the noise model, we thus need the values for the parameters $p$ and $q$ that control the amount and length of the bursts, and the limits for the intervals $\mathscr{A}, \mathscr{B}, \mathcal{O}$, and $\mathscr{Y}$ that affect the behavior of a single burst. If our image is artificial, then the relative variance $\sigma_{\mu}^{2}$ of the multiplicative noise component is also needed.

When forming the test images in this paper (see Section 5.2), the parameter values used were $p=0.0007, q=0.011$, $\mathscr{A}=[160,190], \mathscr{B}=[50,90], \mathscr{O}=[0.3,1.0]$, and $\mathscr{S}=$ $[22,40]$. The relative variance $\sigma_{\mu}^{2}$ for the multiplicative noise in the artificial images was 0.02 . Naturally, the parameter values given here are not the only possibilities but other slightly different parameter values could be used as well. However, the chosen values are suitable to our purposes, and based on our experiments, the values given here may also be used even in quite different situations.

As the selection of the parameter values was based on a detailed analysis of the real remote sensing images, the values can also be used as a starting point for the selection of the parameter values in other cases. That is, when choosing parameter values for some other case, we can start from the values given here, and if we want any changes to the noise characteristics, we can modify the values in a straightforward way to suit other purposes.

For example, if we want less bursts, we can choose a smaller value for $p$, and if we want to increase the number of bursts, we can increase the value of $p$. Likewise, smaller values for $q$ imply longer bursts and larger values for $q$ imply shorter bursts. The overall level of the multiplicative noise can be controlled by decreasing or increasing the relative variance $\sigma_{\mu}^{2}$, and if we want to decrease or increase the average level of the impulse bursts we can, respectively, either decrease or increase the limits of the interval $\mathscr{A}$. Besides, the interval $\mathscr{A}$ can also be shortened or lengthened, which causes, respectively, less or more variations in the average levels of separate bursts.

Decrease in the limits for the amplitude of the harmonic component (i.e., in the interval $\mathscr{B}$ ) implies less variation in a single burst and, conversely, increase implies more variation. The same usually also holds for the limits of the frequency of the harmonic component (i.e., for the interval 0 ) although the periodical nature of the harmonic component may some-

\footnotetext{
${ }^{1}$ Sometimes the half-open intervals $[a, b[$ and $] a, b]$ are also denoted by $[a, b)$ and $(a, b]$, respectively.
} 
times cause odd effects (i.e., aliasing). As above, shorter or longer intervals mean, respectively, less or more variations in the properties of separate bursts. Finally, the weight of the noise component can be controlled by decreasing or increasing the limits for the standard deviation $\sigma_{k}$ (i.e., for the inter$\operatorname{val} \mathscr{Y})$.

Besides variations in the parameter values, other modifications in the noise model are possible as well. For example, instead of multiplicative noise typical for the radar images, additive Gaussian noise typical for optical images can be used.

\section{TRAINING-BASED FILTERING}

In noise removal applications, the task in the training-based design method is to find a filter that transforms the noisy data as close as possible to the desired ideal data. The obtained filter can then be applied to other situations with similar characteristics as well. Several error criteria can be used and the training data can be either natural or artificially generated (see, e.g., $[13,14,15])$.

The filter is usually sought from a specified filter class to keep the optimization reasonably simple. In this paper, we utilize the class of soft morphological filters. This class was selected since we know that the training-based optimized soft morphological filters are able to remove line-type noise efficiently [10]. Moreover, although the optimization of the soft morphological filters is not at all trivial, it can be done in a reasonable time.

\subsection{Soft morphological filters}

Soft morphological filters form a class of stack filters and were introduced to improve the behavior of standard flat morphological filters in noisy conditions [16]. They have many desirable properties, for example, they can be designed to preserve details well [17]. In addition, they are suitable for impulsive or heavy-tailed noise.

The two basic soft morphological operations are soft erosion and soft dilation. Based on them, compound operations can be defined in the usual way.

Definition 5.1. The structuring system $[B, A, r]$ consists of three parameters, finite sets $A$ and $B, A \subseteq B \neq \varnothing$, of $\mathbb{Z}^{2}$, and an integer $r$ satisfying $1 \leq r \leq \max \{1,|B \backslash A|\}$. The set $B$ is called the structuring set, $A$ its (hard) center, $B \backslash A$ its (soft) boundary, and $r$ the order index of its center or the repetition parameter.

The translated set $T_{x}$, where the set $T \subset \mathbb{Z}^{2}$ is translated by $x, x \in \mathbb{Z}^{2}$, is defined by $T_{x}=\{x+t: t \in T\}$. The symmetric set of $T$ is the set $T^{s}=\{-t: t \in T\}$. A multiset is a collection of objects, where the repetition of objects is allowed. For example, $\{1,1,1,2,3,3\}=\{3 \diamond 1,2,2 \diamond 3\}$ is a multiset.

Soft morphological operations transform a signal $X$ : $\mathbb{Z}^{2} \rightarrow \mathbb{R}$ to another signal by the following rules.

Definition 5.2. Soft erosion of $X$ by the structuring system $[B, A, r]$ is denoted by $X \ominus[B, A, r]$ and is defined by $X \ominus$
$[B, A, r](x)=$ the $r$ th smallest value of the multiset $\{r \diamond X(a)$ : $\left.a \in A_{x}\right\} \cup\left\{X(b): b \in(B \backslash A)_{x}\right\}$ for all $x \in \mathbb{Z}^{2}$.

Definition 5.3. Soft dilation of $X$ by the structuring system $[B, A, r]$ is denoted by $X \oplus[B, A, r]$ and is defined by $X \oplus$ $[B, A, r](x)=$ the $r$ th largest value of the multiset $\{r \diamond X(a)$ : $\left.a \in A_{x}\right\} \cup\left\{X(b): b \in(B \backslash A)_{x}\right\}$ for all $x \in \mathbb{Z}^{2}$.

A finite composition of length $p$ of basic soft morphological operations is given by

$$
\begin{array}{r}
\left(\cdots\left(\left(X \otimes_{1}\left[B_{1}, A_{1}, r_{1}\right]\right) \otimes_{2}\left[B_{2}, A_{2}, r_{2}\right]\right) \otimes_{3} \cdots\right) \\
\otimes_{p}\left[B_{p}, A_{p}, r_{p}\right](x),
\end{array}
$$

where $\otimes_{i} \in\{\ominus, \oplus\}$ for all $i \in\{1,2, \ldots, p\}$. Henceforth, we always mean by the term composite filter a finite composition of basic soft morphological filters. Soft opening and soft closing are special cases of composite soft operations. Then, we have a soft erosion-dilation (opening) or dilation-erosion (closing) pair with equal order index values and symmetric structuring sets. If all the structuring sets $B_{i}$ are subsets of the $n \times m$ rectangle, then $n$ and $m$ (or $n \times m$ ) are called the overall dimensions of the corresponding composite filter.

The detail preservation ability, as well as the noise removal capability of a soft morphological filter, depends on the size and shape of its structuring set and on the value of its order index.

\subsection{Training images}

Although there are no analytical criteria for deciding which soft morphological operation (and with which parameters) is the best for some situation, a suitable operation sequence and its parameters can be found using supervised learning methods, for example, simulated annealing and genetic algorithms [10]. Of course, some training set, for which the desired output is known, is needed.

In this paper, we use both artificial images and real satellite images as training images. An artificial test image of size $256 \times 256$ and its three noisy counterparts are presented in Figure 4. The image in Figure 4a is the noise-free test image, the image in Figure $4 \mathrm{~b}$ is corrupted by multiplicative noise only, the image in Figure $4 \mathrm{c}$ is corrupted by impulse bursts only, and the image in Figure 4d is corrupted both by multiplicative noise and by impulse bursts. As can be seen, the test image contains homogeneous regions, large size objects with different shapes, and small size objects also having different shapes, contrasts, and orientations. To simulate the presence of texture in real satellite images, the test image also contains four textural regions with different spatial correlation and statistical properties.

Our desire was also to check whether the soft morphological filters destroy many details while removing the impulse bursts. By comparing the images in Figures 1 and 4, we can see that the structure and general properties of the images are similar enough also for this purpose.

Besides artificial test images, we also used satellite images as training images. Four such training image pairs are shown in Figures 5 and 6 where original satellite images of 


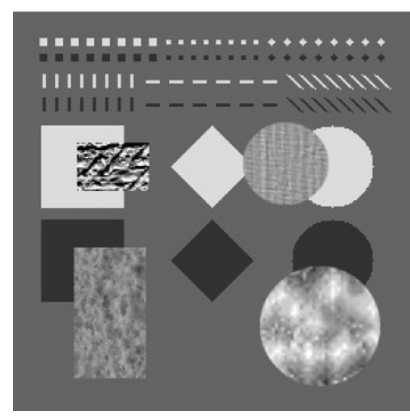

(a)

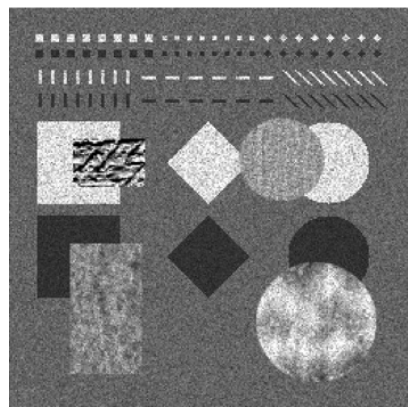

(b)

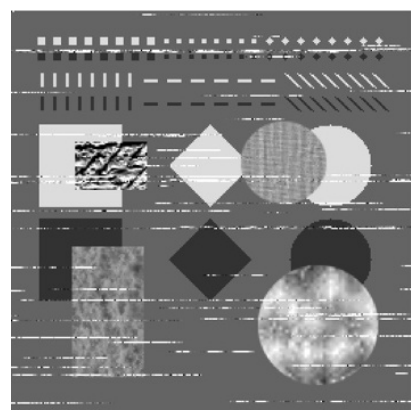

(c)

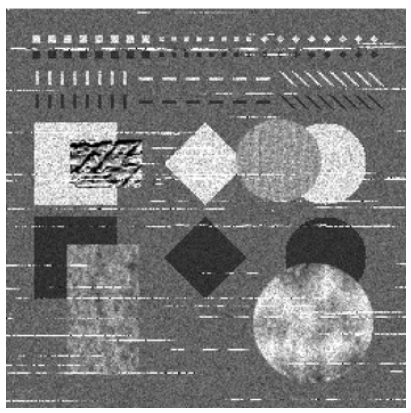

(d)

FIgURE 4: The artificial test images used. (a) The noise-free (i.e., uncorrupted) image. The original image corrupted (b) by multiplicative noise, (c) by impulse bursts, and (d) both by multiplicative noise and by impulse bursts.

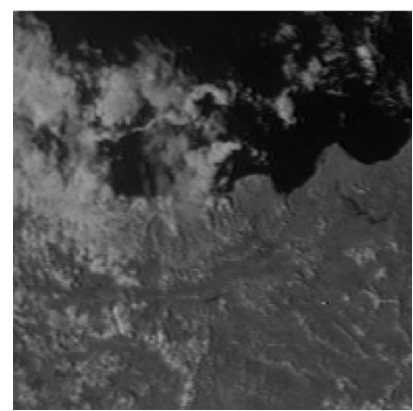

(a)

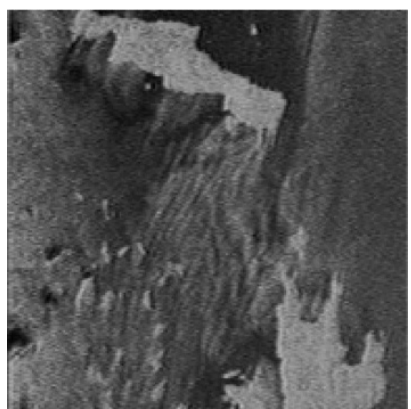

(b)

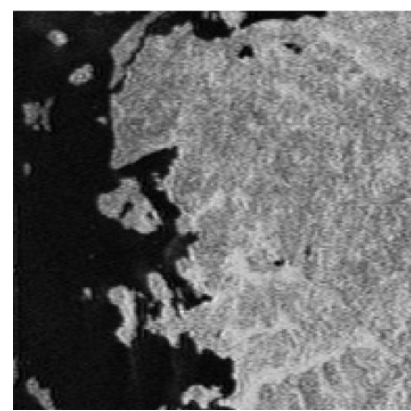

(c)

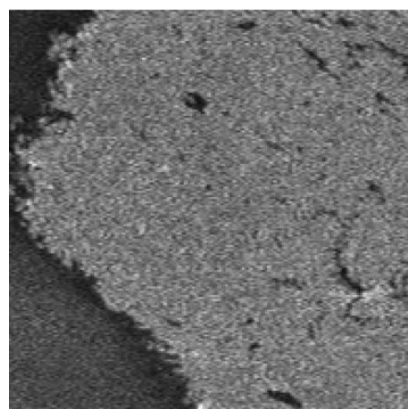

(d)

FigURE 5: Four $192 \times 192$ parts of the original satellite images.

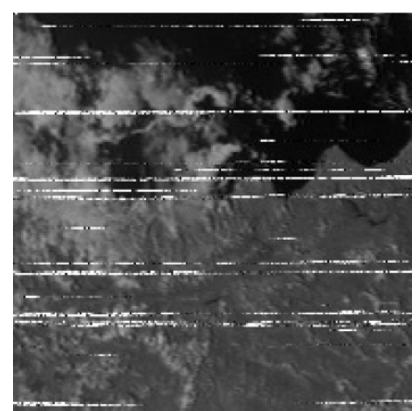

(a)

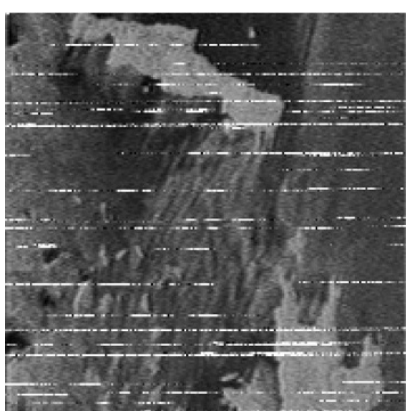

(b)

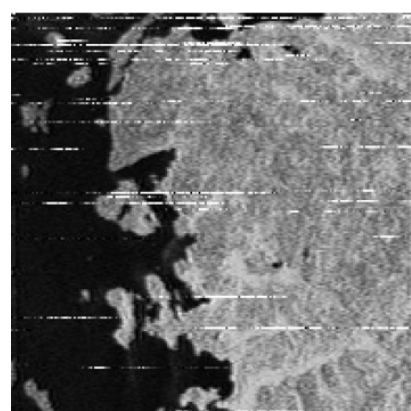

(c)

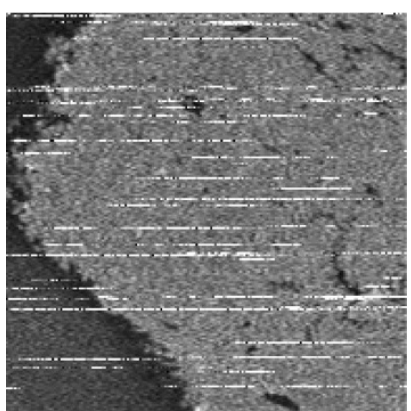

(d)

Figure 6: The images in Figure 5 corrupted by impulse bursts.

size $192 \times 192$ (Figure 5) and their counterparts corrupted by bursts (Figure 6) are represented. The latter images are obtained by corrupting the original images by impulse bursts. A restriction concerning the satellite training images is that, unfortunately, we do not have noise-free test images but all images are corrupted by multiplicative noise. Hence, these images can be used if we try to remove only impulse bursts but they cannot be used if we also try to remove multiplicative noise at the same time.
When forming the test images, the parameter values used in the noise model for the impulse bursts were $\mathscr{A}=$ $[160,190], \mathscr{B}=[50,90], \mathscr{O}=[0.3,1.0]$, and $\mathscr{S}=[22,40]$. The parameter values controlling the amount and length of the impulse bursts were $p=0.0007$ and $q=0.011$ for the test images that contained bursts and $p=0$ and $q=1$ for the test image in Figure $4 \mathrm{~b}$ (in which case no bursts appeared). The relative variance $\sigma_{\mu}^{2}$ for the multiplicative noise was 0.02 for the test images in Figures $4 \mathrm{~b}$ and $4 \mathrm{~d}$ and 0 for the 
other test images (in which cases no multiplicative noise was added).

For technical reasons, we made one technical modification to the noise model when forming the test images in this paper. Namely, the satellite images are often transferred as a group where several images are considered to be one larger image. Thus, although it may seem that one burst continues from the right end of a line to the beginning of the next line, it may be that in reality one burst does not continue from one line to another but, in fact, we have two separate bursts. Hence, we have supposed that if a burst continues from one line to another, the values of the parameters $\alpha_{k}, \beta_{k}, \omega_{k}, \varphi_{k}$, and $\sigma_{k}$ common to a burst are also changed.

\subsection{Optimization}

The optimization methods given in Koivisto et al. [10] allow one to handle impulse bursts in several ways. Basically, there are two different possibilities. We can either try to remove both the impulse bursts and the multiplicative noise at the same time or concentrate to remove only the impulse burst and disregard the multiplicative noise. The latter approach may be useful, for example, if the amount of the multiplicative noise is low.

If we try to remove both the impulse bursts and the multiplicative noise, a straightforward solution is to use a source image that contains both impulse bursts and multiplicative noise and a target image that is free of the bursts and of the multiplicative noise. A suitable training image pair is thus, for example, the image in Figure $4 \mathrm{~d}$ as the source image and the image in Figure $4 \mathrm{a}$ as the target image.

A more refined solution is to employ structural constraints, in which case the target image is again the noise-free image but the source image is the image corrupted by multiplicative noise only. Thus, a suitable training image pair is, for example, the image in Figure $4 \mathrm{~b}$ as the source image and the image in Figure 4a as the target image. The impulse bursts are presented as constraints and an optimal filter is sought provided that the impulse bursts are removed (totally or at least to some extent). This method is more flexible than the straightforward one since we can now control to what extent the impulse bursts should be removed. Unfortunately, this also means that the method needs more tuning, that is, there are more parameters for the user.

Both of the aforementioned methods need a noise-free training image as the target image. Since the real satellite images are in any case corrupted by multiplicative noise, they cannot be used. Unfortunately, only artificial training images can thus be used with these methods.

The other possibility is to optimize the soft morphological operations to remove only impulse bursts (and to preserve details). At the second stage, multiplicative noise can then be suppressed by some conventional technique suited for this purpose, for example, the local statistic Lee filter, the sigma filter, or a combination of them $[4,18,19,20,21]$. In general, the selection of a suitable filter for the postprocessing may depend on the task at hand. However, we can say that the local statistic Lee filter [18] and the locally adaptive schemes
[21], where the local statistic Lee filter is applied only to texture regions, seem to preserve edges, details, and texture features well.

Again, we can use the straightforward solution or we can utilize the structural constraints. In the first case, the training image pair consists of an image corrupted by impulse bursts as the source image and the same image without bursts as the target image. In the latter case, we use the same image as the source and target image. In theory, any images can be used as training images, but in practice, the training images should be such that they incite the filters to preserve details well. The impulse removal is namely not the only goal but the optimal filter should also preserve details well, that is, it is very easy to remove all bursts if we may destroy all details.

Suitable artificial training image pairs in the straightforward solution are thus, for example, the test image corrupted only by the impulse bursts (Figure 4c) as the source image and the noise-free image in Figure $4 \mathrm{a}$ as the target image, or the test image corrupted by impulse bursts and multiplicative noise (Figure $4 \mathrm{~d}$ ) as the source image and the test image corrupted by multiplicative noise (Figure $4 \mathrm{~b}$ ) as the target image. The motivation for the first training image pair is that if we are trying to preserve details and to remove impulse bursts only, then the test images should not contain any other type of noise. The motivation for the latter case is that since impulse bursts usually appear together with multiplicative noise, bursts should also be removed assuming that the images contain multiplicative noise.

The last comment also motivates the use of real satellite images as training images. That is, if we have satellite images that are not corrupted by impulse bursts, they can also be used as training images. Suitable training image pairs are thus also the test images corrupted by impulse bursts (Figure 6) as the source images together with the corresponding original satellite images in Figure 5 as the target images.

If we utilize structural constraints, all aforementioned images that do not contain impulse bursts can be used as the source/target image. Since our aim under the structural constraints is good detail preservation, it may, however, be unreasonable to use test images corrupted heavily by multiplicative noise as the source/target image.

As the error criterion, it is possible to use any criterion that can be calculated using two images as parameters. In this paper, we have used the mean absolute error (MAE) and the mean square error (MSE). Sometimes, the peak signalto-noise ratio

$$
\mathrm{PSNR}=10 \log _{10}\left(255^{2} / \mathrm{MSE}\right)
$$

is also calculated for comparison purposes.

It must be stressed that the goodness of the training concept depends heavily on the practical ingredients such as the sufficiency of the training set and the generalization power of the obtained solution. Experimental tests [10] show that usually a $64 \times 64$ training image is large enough for the training of the soft morphological filters. In this paper, the training images are of size $192 \times 192$ or $256 \times 256$, that is, they are several times larger than a $64 \times 64$ image. Thus, they should be more 
than large enough to prevent overlearning. The experimental results given in Section 6 demonstrate that the designed filter can solve possible new situations in a satisfactory manner.

\section{EXPERIMENTAL RESULTS}

First, we should note that in this paper we call the best filters obtained by our method optimal although there is no absolute guarantee that they are globally optimal.

\subsection{Test case}

The experimental tests reported in this paper are based on the following test cases. The training image pairs are the ones discussed in Sections 5.2 and 5.3. The application images are the ones shown in Figure 1. The optical image in Figure 1d is included for comparison purposes. In each test, an optimal composite operation of length two was sought with overall dimensions $3 \times 3,3 \times 5$ (i.e., 3 columns and 5 rows), and $5 \times 5$. Both nonsymmetric and symmetric structuring sets were used. Note that, in this section, "symmetric structuring set" means that the structuring set is symmetric with respect to the $x$ - and $y$-axes, not with respect to the origin as the symmetric set was defined in Section 5.1.

The length two was selected since the noisy images contain both positive and negative impulsive noise and a single basic soft operation is not able to remove two-sided noise. On the other hand, as the experiments show, two consecutive soft operations are already powerful enough for our purposes.

\subsection{Basic results}

When the $3 \times 3$ window was used, the optimal filters were not able to remove the impulse bursts sufficiently. On the other hand, the filters optimal inside the $3 \times 5$ and $5 \times 5$ windows were already able to remove almost all of the bursts. Hence, the quality of these filters depends on their ability to remove multiplicative noise and preserve details. As the $3 \times 5$ case is a subcase of the $5 \times 5$ case, an optimal composite filter with the overall dimensions $5 \times 5$ naturally outperforms the one with the overall dimensions $3 \times 5$. On the other hand, the optimization is easier with the overall dimensions $3 \times 5$. In practice, the results with the overall dimensions $5 \times 5$ are only slightly better than those with the overall dimensions $3 \times 5$, and the optimization using the overall dimensions $3 \times 5$ is much easier than the optimization using the overall dimensions $5 \times 5$. Hence, in our examples, it is not reasonable to use the overall dimensions $5 \times 5$ but the examples are based mostly on the $3 \times 5$ case.

The results obtained using symmetric structuring sets were usually not as good as those which were achieved without any restrictions (i.e., nonsymmetric structuring sets were also allowed). However, the differences were usually small. The PSNRs obtained by the symmetric structuring systems were usually only $0.1 \mathrm{~dB}$ less than the corresponding values for the nonsymmetric case (see Tables 1 and 2). Since the noise process is symmetric and we cannot make any assumptions about the structure of the application images, it is in any case safe to use symmetric structuring sets. Thus, most of the examples in this paper are also based on the symmetric structuring sets.

The results are at least in the quantitative sense better when using nonsymmetric structuring sets because in soft morphological filtering, the ratio $r /|B \backslash A|$ (i.e., the value of the order index divided by the size of the soft boundary) plays a very important role [10], and with nonsymmetric structuring sets, we have much more possibilities to tune this ratio to be suitable for the optimization task in question, especially when the size of the soft boundary is small. An undesirable side effect is that sometimes this may also lead to slight overlearning. This ratio has much to do with the breakdown point of a basic soft morphological filter [22], and the ratio controls the amount of the impulsive noise that our filters can remove, so that the lower the value for the ratio is, the more impulses will be removed. The optimal value for the ratio is then the highest value such that almost all impulse bursts will be removed.

The optimal filter sequence was usually a soft erosion followed by a soft dilation, as can also be seen from the optimal sequences in Figures 7, 11, and 12. This combination is natural since the impulse bursts were mostly positive. The results obtained by the optimal soft openings were usually almost as good as those obtained using the optimal composite soft operations of length two. This is important since the optimization of soft openings is much easier than the optimization of the composite soft operations of length two.

The error criterion (i.e., the MAE or the MSE) did not seem to have crucial effect in the optimization. The filters optimized under the MSE produced usually visually better results although, in general, the differences were small.

When comparing the optimization schemes, we noticed that by selecting the details in the optimization schemes in a suitable manner, all schemes were able to produce good results. The suitability of some optimization scheme thus depends much on whether we want to emphasize the burst removal capability or the detail preservation ability of the resulting filter.

\subsection{Bursts and multiplicative noise}

In this section, we study the experiments where we remove both impulse bursts and multiplicative noise at the same time. Both the straightforward optimization and the structural constraints are employed.

The structuring systems of the operation sequence optimized utilizing the straightforward method are given in Figure 7a. The sequence was found under the MSE and inside the $3 \times 5$ window. Symmetric structuring sets were used. The source image was the artificial image corrupted both by the impulse bursts and by the multiplicative noise (Figure $4 \mathrm{~d}$ ) and the target image was the noise-free image in Figure 4a. Clearly, both operations have their own task. The first operation is a soft erosion with large structuring set. It removes the bursts, and the large structuring set guarantees that the bursts are removed with efficiency. The second operation is a small soft dilation that removes the negative parts of the bursts and suppresses multiplicative noise. 
TABLE 1: The MSEs (and the corresponding PSNRs) between the target training images and the source training images filtered by the optimal filters with the overall dimensions $3 \times 5$ and the symmetric and nonsymmetric structuring sets. The filters were trained to remove impulse bursts only.

\begin{tabular}{lcccc}
\hline & & MSE & \\
\hline Source image & Target image & Original & Symmetric & No restrictions \\
\hline Figure 4c & Figure 4a & 483.4 & 85.2 & 80.6 \\
Figure 4d & Figure 4b & 490.8 & 147.9 & 145.6 \\
Figure 6a & Figure 5a & 1059.4 & 42.4 & 40.3 \\
Figure 6b & Figure 5b & 1069.7 & 43.7 & 43.6 \\
Figure 6c & Figure 5c & 460.7 & 61.5 & 61.5 \\
Figure 6d & Figure 5d & 860.8 & 122.0 & 121.5 \\
\hline & & PSNR & & No restrictions \\
\hline Source image & Target image & Original & Symmetric & 29.1 \\
\hline Figure 4c & Figure 4a & 21.3 & 28.8 & 26.5 \\
Figure 4d & Figure 4b & 21.2 & 26.4 & 32.0 \\
Figure 6a & Figure 5a & 17.9 & 31.9 & 31.7 \\
Figure 6b & Figure 5b & 17.8 & 31.7 & 30.2 \\
Figure 6c & Figure 5c & 21.5 & 30.2 & 27.3 \\
Figure 6d & Figure 5d & 18.8 & 27.3 & \\
\hline
\end{tabular}

TABLE 2: The MSEs (and the corresponding PSNRs) between the target training image and the source training image filtered by the optimal filters with the overall dimensions $3 \times 5$ and the symmetric and nonsymmetric structuring sets. The filters were trained to remove both impulse bursts and multiplicative noise. Note that different methods utilize different source images.

\begin{tabular}{|c|c|c|c|}
\hline \multicolumn{4}{|c|}{ MSE } \\
\hline Optimization method & Original & Symmetric & No restrictions \\
\hline Straightforward optimization & 745.5 & 190.0 & 187.7 \\
\hline Structural constraints & 274.0 & 162.3 & 160.6 \\
\hline \multicolumn{4}{|c|}{ PSNR } \\
\hline Optimization method & Original & Symmetric & No restrictions \\
\hline Straightforward optimization & 19.4 & 25.3 & 25.4 \\
\hline Structural constraints & 23.8 & 26.0 & 26.1 \\
\hline
\end{tabular}

Figure $8 \mathrm{a}$ shows the resulting image when the noisy image in Figure $4 \mathrm{~d}$ is filtered using the optimal filter in Figure 7a. As can be seen, the image in Figure 8a is a little blurred and some small details are lost. However, practically, all impulse bursts have disappeared and the texture as well as most of the details are preserved.

Figure 9 illustrates what happens when the filter sequence in Figure $7 \mathrm{a}$ is applied to the real satellite images given in Figure 1. Again, almost all impulse bursts have disappeared and small distortion has appeared. It is also worth mentioning that although the training image in our case study was based on radar images (i.e., multiplicative noise), the obtained optimal filter also works well with the optical image in Figure 9d that was originally corrupted instead of multiplicative noise by additive noise. Hence, the obtained filter can be applied to a variety of different satellite images.

When the structural constraints were used together with the requirement that all impulse bursts must be removed, the resulting images were somewhat blurred. Hence, if structural constraints are used, it is advisable to allow that a small portion of impulse bursts may remain after the filtering. Naturally, the requirement to which extent the bursts must be removed can be used to control the detail preservation ability and the impulse removal capability of the optimal filter in other ways as well.

Figure $7 \mathrm{~b}$ shows the structuring systems of the operation sequence optimized utilizing the structural constraints. Again, the sequence with symmetric structuring sets was found under the MSE and with the overall dimensions $3 \times 5$. The source image was the artificial test image corrupted by the multiplicative noise (Figure $4 \mathrm{~b}$ ) and the target image was the noise-free image in Figure 4a. The impulse bursts were presented as constraints and the optimal filter was sought provided that almost all (however, not all) of the impulse bursts are removed.

As can be seen from the optimal structuring systems, the first operation (soft erosion) is clearly concentrated on burst removal and the second operation (soft dilation) on the 


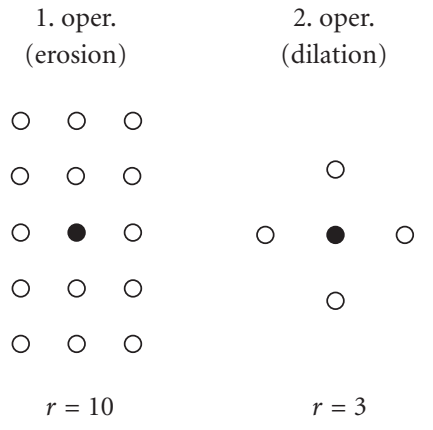

(a)

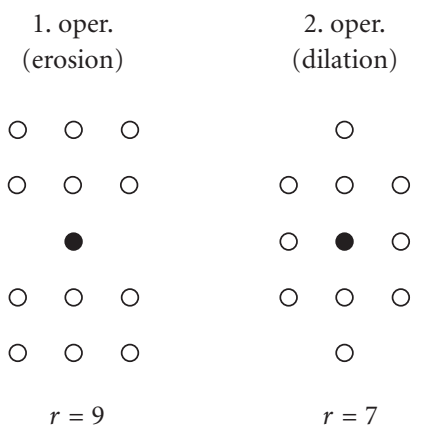

(b)

FIGURE 7: The (symmetric) structuring systems of the soft operation sequences optimized to remove both impulse bursts and multiplicative noise utilizing (a) straightforward optimization and (b) structural constraints $(\bullet=$ the hard center $=$ the origin, $\circ=$ the soft boundary, and $r=$ the order index).

removal of multiplicative noise. Although the optimal structuring systems are not the same as those obtained using the straightforward optimization, they are, however, quite similar. In both cases, the second operation focuses on the multiplicative noise and the first operation is a soft erosion with large structuring set, which is suitable for the burst removal. Moreover, the ratios $r /|B \backslash A|$ do not differ much. For the structuring systems obtained using the straightforward optimization, they are 0.71 and 0.75 , and with the structural constraints, they are 0.75 and 0.7 . Hence, both operation sequences should perform much in the same way.

Figure $8 \mathrm{~b}$ shows the image that is obtained by filtering the image corrupted by the impulse bursts and the multiplicative noise (Figure 4d) using the filter sequence in Figure 7b. As can be seen, the optimal filter removes bursts and multiplicative noise well but at the same time some small, especially horizontal, details are lost. When comparing the images in Figures $8 \mathrm{a}$ and $8 \mathrm{~b}$, we notice that the filter obtained using the structural constraints removes better impulse bursts than the filter obtained by the straightforward method. Unfortunately, at the same time, it also destroys more details.

As can be seen from the images in Figure 10, the aforementioned phenomenon also appears when the satellite

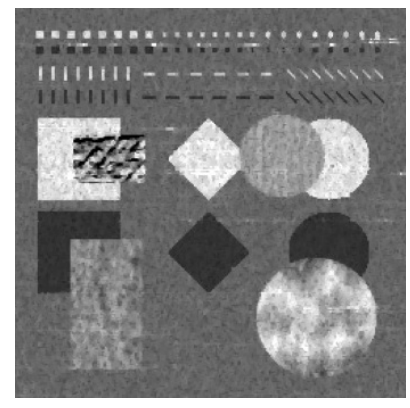

(a)

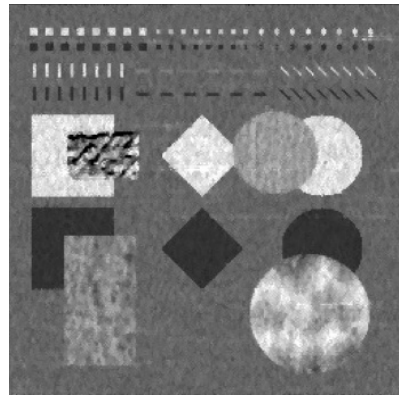

(b)
Figure 8: The artificial test image in Figure 4d filtered by the optimal symmetric composite soft operation of length two. The filters were optimized to remove both impulse bursts and multiplicative noise using (a) straightforward optimization and (b) structural constraints.

images are filtered by the filter sequence in Figure $7 \mathrm{~b}$. That is, only few impulse bursts remain but some very small details have disappeared. Again, the obtained filter also works well with the optical image in Figure 10d.

\subsection{Burst removal}

Next, we concentrate on the removal of the impulse bursts. In the tests, four satellite images and one artificial image (both with and without multiplicative noise) were used as the training images. Some of the optimal symmetric structuring systems with overall dimensions $3 \times 5$ found under the MSE are shown in Figures 11 and 12. Again, the optimal filter sequences were soft erosions followed by soft dilations. The filters in Figure 11 were optimized utilizing the satellite training images, and the filters in Figure 12 were obtained using the artificial training images. The target images were thus the satellite images in Figure 5 and the artificial images in Figures $4 \mathrm{a}$ and $4 \mathrm{~b}$, and the source images were the target images corrupted by impulse bursts, that is, the satellite images in Figure 6 and the artificial images in Figures $4 \mathrm{c}$ and $4 \mathrm{~d}$, respectively.

Although not identical, the optimal structuring systems are quite similar. They also have much in common with the optimal structuring systems in Section 6.3. Again, the first operation (soft erosion) is the one that removes the bursts. Moreover, the structuring systems of the first operation are nearly alike. The second operation (soft dilation) is in all cases very weak and its role is to remove the negative parts of the bursts and to correct the bias that the first operation causes.

For all optimal filters, the value of the order index of the second operation is equal to the size of the soft boundary, which means that only a few changes upwards will be made. The size of the soft boundary of the second operation of the optimal operation sequence depends in a straightforward way on the amount of the details in the training image. That is, the more texture the training image has, the larger structuring set we have for the second operation. The 


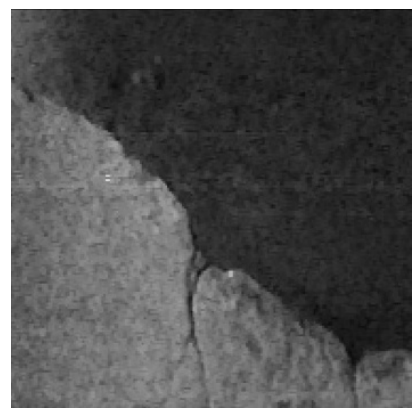

(a)

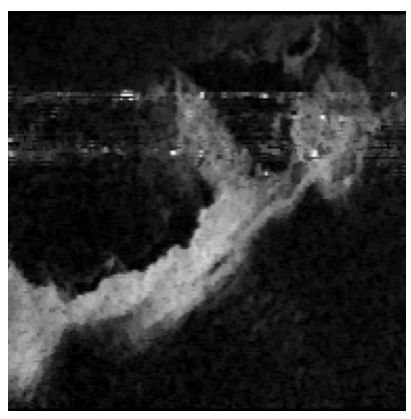

(b)

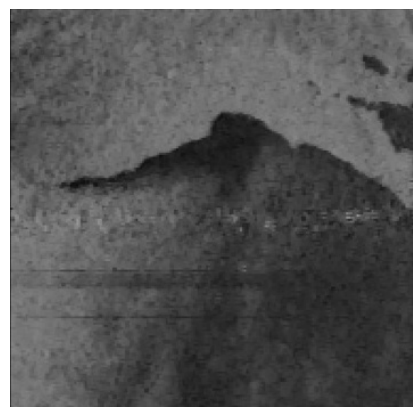

(c)

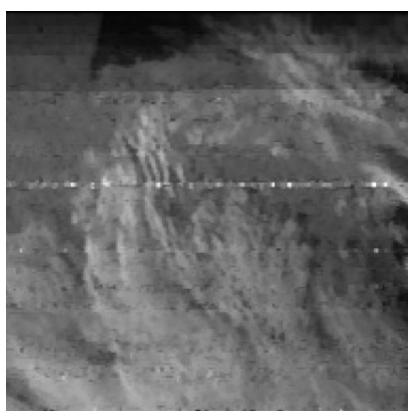

(d)

FIGURE 9: The original satellite images in Figure 1 filtered by the optimal symmetric composite soft operation of length two (see Figure 7a) that was optimized using the straightforward optimization.

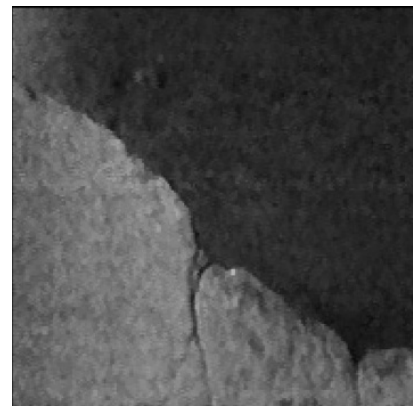

(a)

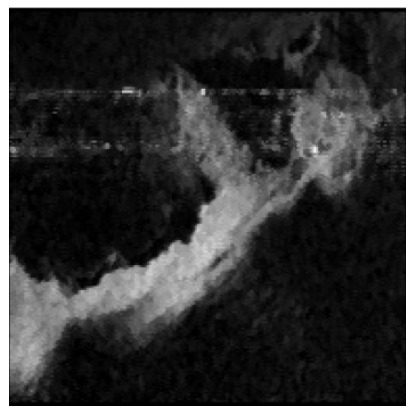

(b)

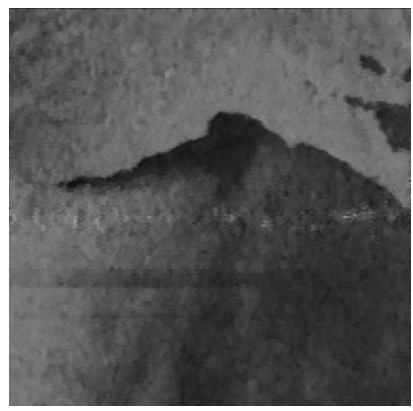

(c)

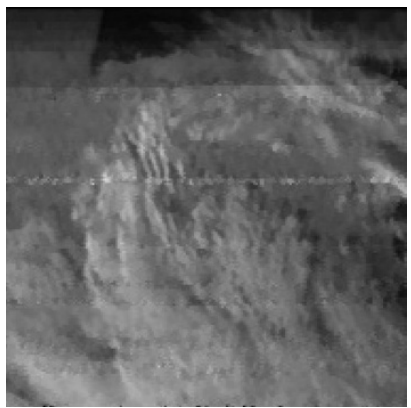

(d)

FIGURE 10: The original satellite images in Figure 1 filtered by the optimal symmetric composite soft operation of length two (see Figure $7 \mathrm{~b}$ ) that was optimized using the structural constraints.

reason is that a large structuring set means now less changes upwards. Hence, a large structuring set also produces better detail preservation which is useful when the training image contains a lot of texture.

In all cases, the optimal filters could remove impulse bursts with efficiency, as can also be seen from the MSE and PSNR values in Table 1. Since the amount of the bursts is about the same in all cases, the differences in the original errors are quite much explained by the fact that the general level of the background varies from image to image. That is, the darker the background is, the larger values for the MSE we have. The substantial improvement for the test images in Figures $5 \mathrm{a}$ and $5 \mathrm{~b}$ is explained by the smaller amount of texture in these images. The smallest improvement was achieved when the artificial images corrupted by the multiplicative noise were used as the training images. Then, the target image contained so many "details" (i.e., details, texture, and multiplicative noise) that the optimal filter was not anymore able to remove bursts with full efficiency. The results obtained utilizing structural constraints where in coherence with the results reported here.

Visually, the differences between different schemes were again quite small. For comparison purposes, Figure 13 shows two images that are obtained by filtering the artificial test image corrupted both by the multiplicative noise and the impulse bursts (Figure $4 \mathrm{~d}$ ) using the found optimal filters. The image in Figure 13a is the result when the image in Figure 4d is filtered by the operation sequence in Figure 11a and the image in Figure 13b is the result when the same image is filtered by the operation sequence in Figure 12a. That is, the first filter was optimized with the satellite image in Figure 6a as the source image and the satellite image in Figure $5 \mathrm{a}$ as the target image, and the second filter was optimized with the artificial image in Figure $4 \mathrm{c}$ as the source image and the noise-free artificial image in Figure $4 \mathrm{a}$ as the target image.

As can be seen, the filter that was optimized using the satellite images as training images can remove practically all impulse bursts. Unfortunately, at the same time, some small details are lost. The filter that was optimized using the artificial noise-free image as the target image can preserve details very well but a small amount of bursts is remaining. The reason for the latter case is that since we do not have multiplicative noise in the training images, the optimal filter can put more emphasis on the detail preservation than the optimal filters in the other cases. Thus, the resulting filter has the best detail preservation ability of the filters. 


$$
\begin{array}{lc}
\text { 1. oper. } & \text { 2. oper. } \\
\text { (erosion) } & \text { (dilation) }
\end{array}
$$

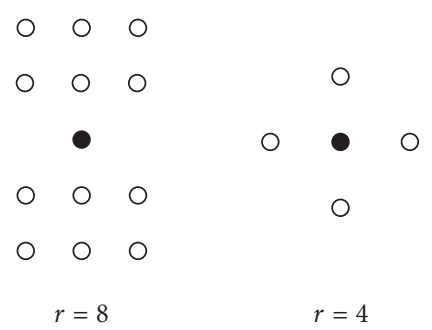

\begin{tabular}{|c|c|c|c|}
\hline \multicolumn{2}{|c|}{$\begin{array}{l}\text { 1. oper. } \\
\text { (erosion) }\end{array}$} & \multicolumn{2}{|c|}{$\begin{array}{l}\text { 2. oper. } \\
\text { (dilation) }\end{array}$} \\
\hline $0 \quad 0$ & 0 & & 0 \\
\hline $0 \quad 0$ & 0 & 0 & 00 \\
\hline - & & 0 & - \\
\hline 0 & 0 & 0 & O \\
\hline $0 \quad 0$ & 0 & & 0 \\
\hline$r=9$ & & & $r=10$ \\
\hline
\end{tabular}

(a)

(b)

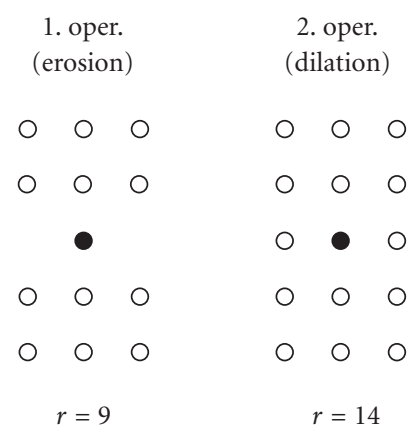

(c)

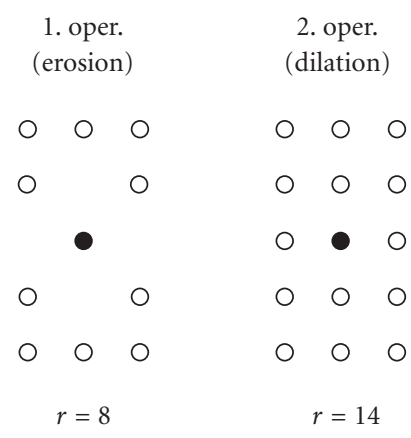

(d)

FIGURE 11: The (symmetric) structuring systems of the soft operation sequences optimized using the satellite images in Figures (a) $6 \mathrm{a}$ and $5 a$, (b) $6 b$ and $5 b$, (c) $6 c$ and $5 c$, (d) $6 d$ and $5 d$ as the training images $(\bullet=$ the hard center $=$ the origin, $\circ=$ the soft boundary, and $r=$ the order index).

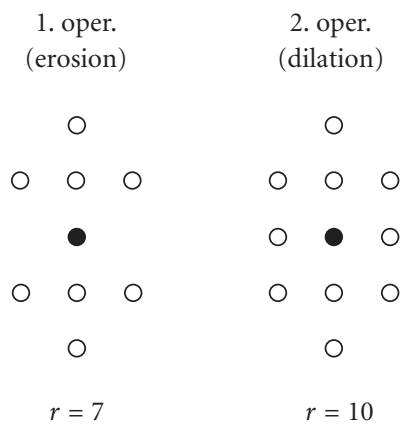

(a)

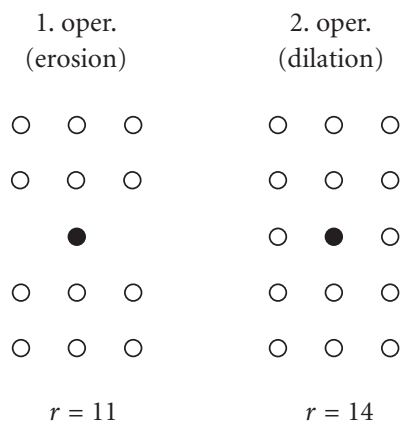

(b)

FIGURE 12: The (symmetric) structuring systems of the soft operation sequences optimized using the artificial images in Figures (a) $4 \mathrm{c}$ and $4 \mathrm{a}$, and (b) $4 \mathrm{~d}$ and $4 \mathrm{~b}$ as the training images $(\bullet=$ the hard center $=$ the origin, $\circ=$ the soft boundary, and $r=$ the order index $)$.

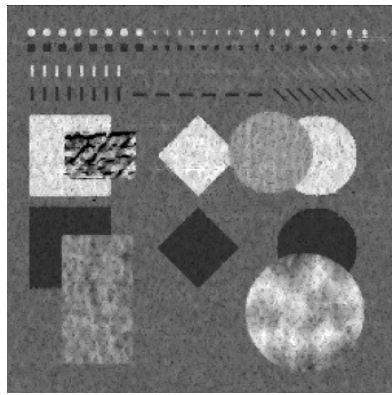

(a)

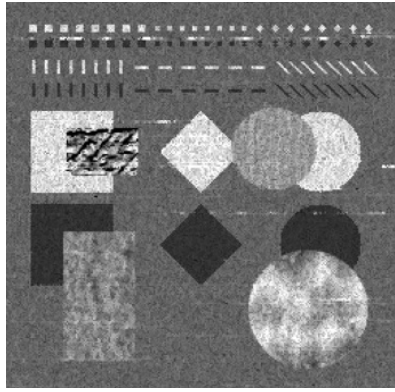

(b)
FIgURE 13: The artificial test image in Figure $4 \mathrm{~d}$ filtered by the optimal symmetric composite soft operation of length two. The filters were optimized to remove impulse bursts only using (a) the satellite training images in Figures $6 a$ and $5 a$, and (b) the artificial training images in Figures $4 \mathrm{c}$ and $4 \mathrm{a}$.

Naturally, most of the multiplicative noise remains in the images but as we already noted, if there is a need, the multiplicative noise can be removed by postprocessing filters. Experimental tests show that with these images, a suitable postprocessing filter can improve the PSNRs about $1.5 \mathrm{~dB}$.

Figure 14 shows what happens when the burst removal 


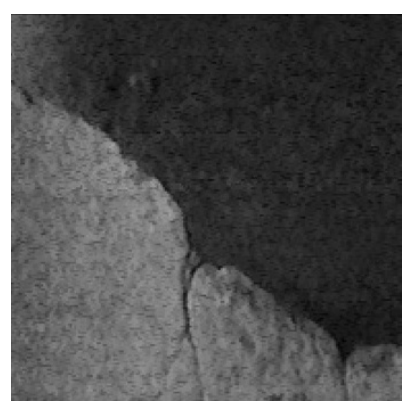

(a)

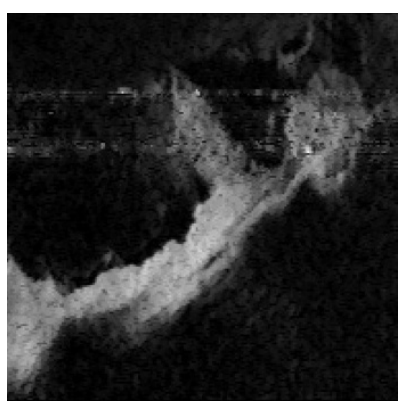

(b)

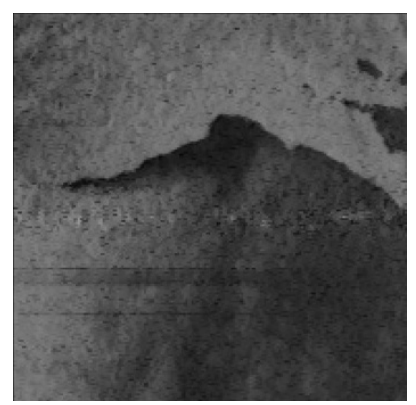

(c)

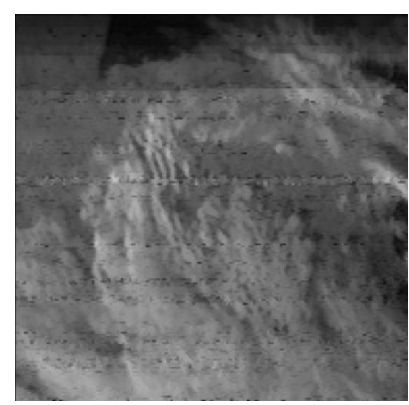

(d)

FIGURE 14: The original satellite images in Figure 1 filtered by the optimal symmetric composite soft operation of length two (see Figure 11a) that was optimized using the satellite images in Figures $6 \mathrm{a}$ and $5 \mathrm{a}$ as the training image pair.

method is applied to the real satellite images. The utilized operation sequence was the one shown in Figure 11a, that is, the one optimized using the satellite image in Figure $6 \mathrm{a}$ as the source image and the satellite image in Figure $5 \mathrm{a}$ as the target image. As can be seen, practically, all bursts have disappeared but most of the negative noise remains, which can be seen as black dots in the images. When compared to images in Figures 9 and 10 , we can see that the results are quite similar. Less bursts and more details remain but, in contrast, the images contain more black dots.

\subsection{Comparison tests}

We also compared the obtained filters to existing filters. The chosen comparison filters were the median filter, the centerweighted median (CWM) filter, and the Wilcoxon filter that are either robust or should perform otherwise efficiently in this kind of situations $[3,5,23]$ (for the definition of the filters, see, e.g., $[3,5])$. For all filters, different window sizes were tested. The quantitative results of the comparison are shown in Table 3. For the median filter and the CWM filter with 3 as the center weight (CWM-3), the best results with respect to the MSE criterion were obtained using the $3 \times 3$ window. For the CWM filter with 5 as the center weight (CWM-5) and for the Wilcoxon filter, the best results were obtained using the $3 \times 5$ window.

The corresponding MSE and PSNR values between the image in Figure $4 \mathrm{a}$ and the image in Figure $4 \mathrm{~d}$ filtered by the filters optimized to remove both the impulse bursts and the multiplicative noise are 187.7 and 25.4 for the operation sequence optimized using the straightforward optimization and 193.3 and 25.3 for the operation sequence optimized using the structural constraints. When compared to the results shown in Table 3, we can see that the methods discussed in this paper clearly outperform the comparison filters. Visually, the resulting images were in coherence with the quantitative results.

Finally, we compared our methods to methods where the idea is first to detect the corrupted pixels and then to replace the corresponding values by new values usually by taking, in some way, into account the neighboring pixel values for which the bursts have not been detected. However, for ex- ample, the method proposed by Abreu et al. [8] did not detect impulse bursts satisfactorily and due to this, quite many bursts were remaining after the filtering. Moreover, since only the values of the detected samples are changed, most of the multiplicative noise remains in the image. Taken all together, this results in MSE value 366.5 (PSNR $22.5 \mathrm{~dB}$ ), which is even worse than that with the other comparison methods.

We also tested the method of primary local recognition proposed by Dolia et al. [9]. The method was introduced to detect spikes in images corrupted by mixed multiplicative and impulsive noise. However, this method also failed and produced a quite low percentage of correctly detected spikes.

We must admit that the burst detection methods may produce good results in some specific cases. Especially, if the amount of the bursts and other noise in the image is low, we can avoid unnecessary filtering if we first detect the bursts. This is also how the soft morphological filters work, that is, if the filter does not think that a sample is an impulse, then no changes, or at least only small changes, will be done. A future research topic is to improve the detection methods so that they also work well with bursts.

\subsection{Concluding remarks}

An effective filtering method with good detail preservation properties is also a desideratum when impulse bursts are removed. Unfortunately, it is difficult to design a filter that at the same time both removes bursts with efficiency and preserves details well. The more emphasis is laid on the detail preservation, the more bursts tend to stay after the filtering.

The filter design method used in this paper offers several ways to control the above dilemma. Utilizing the structural constraints, we can first set a limit to which extent the bursts should be removed and then find the optimal filter supposing that the bursts are removed up to that limit. In the straightforward optimization, we can control the detail preservation ability of the optimal filter by selecting training images that contain different amounts of details. The burst removal, on the other hand, can be controlled by varying the amount of the bursts in the training images. Large amounts of bursts ensure that the method is robust but small burst amounts produce better detail preservation. 
TABLE 3: The MSEs (and the corresponding PSNRs) between the image in Figure 4d filtered by some nonlinear filters and the image in Figure 4a.

\begin{tabular}{lcccccc}
\hline & \multicolumn{2}{c}{$3 \times 3$ window } & \multicolumn{2}{c}{$3 \times 5$ window } & \multicolumn{2}{c}{$5 \times 5$ window } \\
Filter & MSE & PSNR & MSE & PSNR & MSE & PSNR \\
\hline Original error & 745.5 & 19.4 & 745.5 & 19.4 & 745.5 & 19.4 \\
Median & 242.9 & 24.3 & 283.9 & 23.6 & 331.1 & 22.9 \\
CWM-3 & 216.9 & 24.7 & 237.5 & 24.4 & 283.0 & 23.6 \\
CWM-5 & 431.0 & 21.8 & 202.4 & 25.1 & 255.0 & 24.1 \\
Wilcoxon & 357.8 & 22.6 & 336.5 & 22.9 & 342.1 & 22.8 \\
\hline
\end{tabular}

All aforementioned strategies can also be used if we want to remove impulse bursts only. In addition, the error criteria used have, at least up to some extent, similar effects. For instance, the remaining bursts affect the MSE more than the MAE.

Naturally, the training-based design method also has some restrictions. Although experimental tests show that soft morphological filters learn to remove the type of noise that is in the training image, the training image pair is in any case needed. Moreover, the noise in the training image should be similar to that in the application images.

\section{CONCLUSION}

The characteristics of impulse bursts in remote sensing images were analyzed and methods for the removal of the bursts were discussed. It was shown through experiments that the presented methods can remove impulse bursts and multiplicative (or additive) noise with efficiency and at the same time preserve details well. As a case study, composite soft morphological filters were utilized.

The design methods used also allow us to emphasize different aspects of the filtering task, for example, detail preservation or noise removal. Although our test case was quite limited, the method can easily be applied to other cases as well.

\section{REFERENCES}

[1] J. A. Richards, Remote Sensing Digital Image Analysis. An Introduction, Springer-Verlag, Berlin, Germany, 1994.

[2] K.-H. Szekielda, Satellite Monitoring of the Earth, John Wiley \& Sons, New York, NY, USA, 1989.

[3] J. Astola and P. Kuosmanen, Fundamentals of Nonlinear Digital Filtering, CRC Press, Boca Raton, Fla, USA, 1997.

[4] V. P. Melnik, Nonlinear locally adaptive techniques for image filtering and restoration in mixed noise environment, Thesis for the Degree of Doctor of Technology, Tampere University of Technology, Tampere, Finland, March 2000.

[5] I. Pitas and A. N. Venetsanopoulos, Nonlinear Digital Filters: Principles and Applications, Kluwer Academic, Boston, Mass, USA, 1990.

[6] "NOAA Polar Orbiter Data User's Guide," NOAA Publications and Technical Reports, http://www2.ncdc.noaa.gov/docs/ podug/index.htm.

[7] V. V. Lukin, N. N. Ponomarenko, P. Kuosmanen, and J. Astola, "Modified sigma filter for processing images corrupted by multiplicative and impulsive noise," in Proc. 8th European Signal Processing Conference, vol. 3, pp. 1909-1912, Trieste, Italy, September 1996.

[8] E. Abreu, M. Lightstone, S. Mitra, and K. Arakawa, "A new efficient approach for the removal of impulse noise from highly corrupted images," IEEE Trans. Image Processing, vol. 5, no. 6, pp. 1012-1025, 1996.

[9] A. N. Dolia, A. Burian, V. V. Lukin, C. Rusu, A. A. Kurekin, and A. A. Zelensky, "Neural network application for primary local recognition and nonlinear adaptive filtering of images," in Proc. 6th IEEE Conference on Electronics, Circuits and Systems, vol. 2, pp. 847-850, Pafos, Cyprus, September 1999.

[10] P. Koivisto, H. Huttunen, and P. Kuosmanen, "Training-based optimization of soft morphological filters," Journal of Electronic Imaging, vol. 5, no. 3, pp. 300-322, 1996.

[11] J. S. Bendat and A. G. Piersol, Random Data: Analysis and Measurement Procedures, John Wiley \& Sons, New York, NY, USA, 1986.

[12] J. R. Norris, Markov Chains, Number 2 in Cambridge Series on Statistical and Probabilistic Mathematics. Cambridge University Press, Cambridge, UK, 1998.

[13] J.-H. Lin, T. M. Sellke, and E. J. Coyle, "Adaptive stack filtering under the mean absolute error criterion," IEEE Trans. Acoustics, Speech, and Signal Processing, vol. 38, no. 6, pp. 938-954, 1990.

[14] H. Longbotham and D. Eberly, "The WMMR filters: a class of robust edge enhancers," IEEE Trans. Signal Processing, vol. 41, no. 4, pp. 1680-1685, 1993.

[15] I. Tăbuş, D. Petrescu, and M. Gabbouj, "A training framework for stack and Boolean filtering-fast optimal design procedures and robustness case study," IEEE Trans. Image Processing, vol. 5, no. 6, pp. 809-826, 1996.

[16] L. Koskinen and J. Astola, "Soft morphological filters: a robust morphological filtering method," Journal of Electronic Imaging, vol. 3, no. 1, pp. 60-70, 1994.

[17] P. Kuosmanen and J. Astola, "Soft morphological filtering," Journal of Mathematical Imaging and Vision, vol. 5, no. 3, pp. 231-262, 1995.

[18] J.-S. Lee, "Speckle analysis and smoothing of synthetic aperture radar images," Computer Vision, Graphics and Image Processing, vol. 17, no. 1, pp. 24-32, 1981.

[19] J.-S. Lee, "Digital image smoothing and the sigma filter," Computer Vision, Graphics and Image Processing, vol. 24, no. 2, pp. 255-269, 1983.

[20] V. V. Lukin, N. N. Ponomarenko, L. Y. Alekseyev, V. P. Melnik, and J. Astola, "Two-stage radar image despeckling based on local statistic Lee and sigma filtering," in Nonlinear Image Processing and Pattern Analysis XII, E. R. Dougherty and J. Astola, Eds., vol. 4304 of SPIE Proceedings, pp. 106-117, San Jose, Calif, USA, January 2001.

[21] V. V. Lukin and O. V. Tsymbal, "MM-band radar image filtering with texture information preservation," in Proc. 4th URSI 
International Kharkov Symposium "Physics and Engineering of Millimeter and Sub-Millimeter Waves”, vol. 1, pp. 435-437, Kharkov, Ukraine, June 2001.

[22] P. Koivisto, H. Huttunen, and P. Kuosmanen, "Breakdown points and optimal soft morphological filtering," in Proc. 1996 IEEE Digital Signal Processing Workshop, pp. 490-493, Loen, Norway, September 1996.

[23] R. J. Crinon, "The Wilcoxon filter: a robust filtering scheme," in Proc. IEEE Int. Conf. Acoustics, Speech, Signal Processing, vol. 2, pp. 668-671, Tampa, Fla, USA, March 1985.

Pertti Koivisto received the M.S. and Licentiate degrees in mathematics from the University of Tampere, Finland, in 1984 and 1999, respectively. He received the Ph.D. degree in signal processing from the Tampere University of Technology in 2000. Since 1981, he has held various teaching and research positions in mathematics and signal processing at the University of Tampere and at the Tampere University of Technology.

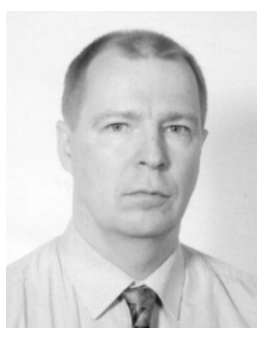
Currently, he is a Senior Researcher at the Institute of Signal Processing, Tampere University of Technology. His main research interests include nonlinear signal and image processing and combinatorial optimization.

Jaakko Astola received his B.S., M.S., Licentiate, and Ph.D. degrees in mathematics (specializing in error-correcting codes) from Turku University, Finland, in 1972, 1973, 1975, and 1978, respectively. From 1976 to 1977 , he was with the Research Institute for Mathematical Sciences of Kyoto University, Kyoto, Japan. Between 1979 and 1987, he was with the Department of Information Technology, Lappeenranta Univer-

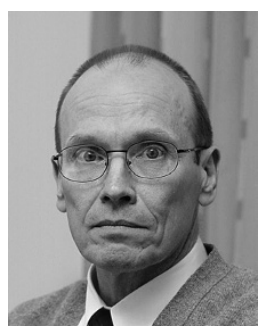
sity of Technology, Lappeenranta, Finland, holding various teaching positions in mathematics, applied mathematics, and computer science. In 1984, he worked as a visiting Scientist at Eindhoven University of Technology, the Netherlands. From 1987 to 1992, he was an Associate Professor of applied mathematics at Tampere University, Tampere, Finland. From 1993, he has been Professor of Signal Processing and Director of Tampere International Center for Signal Processing, leading a group of about 60 scientists and was nominated Academy Professor by the Academy of Finland (20012006). His research interests include signal processing, coding theory, spectral techniques, and statistics. Dr. Astola is a fellow of the IEEE.

Vladimir Lukin was born in 1960 in Belarus (fSU). He graduated in 1983 from the Faculty of Radioelectronic Systems, Kharkov Aviation Institute (now National Aerospace University), Kharkov, Ukraine, and received the Diploma with Honours in radioengineering. Since then, he has been with the Department of Transmitters, Receivers, and Signal Processing of the same faculty. He received Candidate of Technical Science

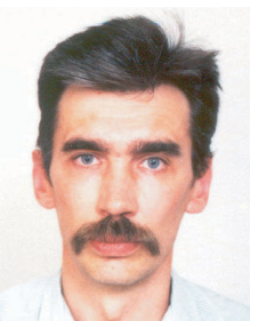
Diploma in radioengineering in 1988 and Senior Researcher Diploma in 1991. In the academic years 1992/1993; he was for 5 months a visiting Researcher at Northern Jiaotong University (Beijing, China). Since 1995, he has been in cooperation with
Tampere University of Technology (Institute of Signal Processing and TICSP). Since 1996, he has been in the Program Committee of Nonlinear Image Processing Conference, SPIE Symposium Photonics West in San Jose, USA. Since 1989, he has been ViceChairman of the Department of Transmitters, Receivers, and Signal Processing. He has published more than 150 journal and conference papers in Ukraine, USA, Finland, Russia, and so forth. More than 70 of them are in English.

Vladimir Melnik was born in 1966 in Khmelnitsky, Ukraine. He graduated from the Kharkov Aviation Institute, Kharkov, Ukraine, in 1992 and received the M.S. degree in electrical engineering. Between 1992 and 1998, he was a Researcher and Senior Researcher at the same institute at the Department of Transmitting and Receiving Devices. In 1997, he received the Candidate of Technical Science degree from the Kharkov Aviation Institute. From 1998 to 2000, he was with the Signal Processing Laboratory at the Tampere University of Technology, Tampere, Finland, where he received the degree of Doctor of Technology in 2000. From 2001, he is working at Nokia, Espoo, Finland. His research interests include image and signal processing and their applications.

Oleg Tsymbal born in 1974, graduated from the National Aerospace University, Kharkov, Ukraine, in 1998 and received the Diploma of Computer Science. He is currently completing his work toward the degree of Candidate of Technical Science at the National Aerospace University in radar image processing. His research interests include digital image processing and radar data interpretation.
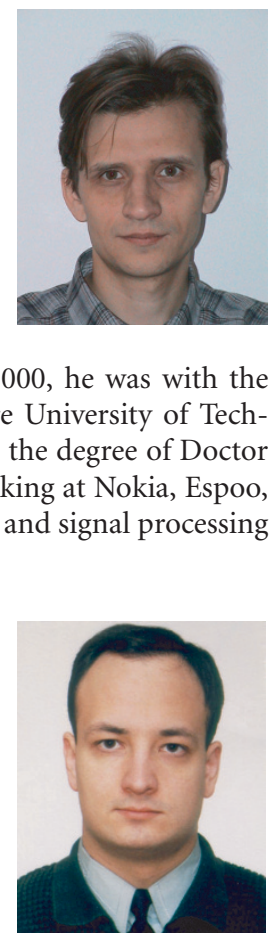